PROCEEDINGS OF THE

AMERICAN MATHEMATICAL SOCIETY

Volume 137, Number 12, December 2009, Pages 3985-3990

S 0002-9939(09)09977-8

Article electronically published on July 1, 2009

\title{
MULTISECANT SUBSPACES TO SMOOTH PROJECTIVE VARIETIES IN ARBITRARY CHARACTERISTIC
}

\author{
ATSUSHI NOMA \\ (Communicated by Ted Chinburg)
}

\begin{abstract}
Let $X \subseteq \mathbb{P}^{N}$ be a projective variety of dimension $n \geq 1$, degree $d$, and codimension $e$, not contained in any hyperplane, defined over an algebraically closed field $\mathbb{k}$ of arbitrary characteristic. We show that if a $k$ dimensional linear subspace $M$ meets $X$ at the smooth locus such that $X \cap M$ is finite and locally lies on a smooth curve, then the length $l(X \cap M)$ does not exceed $d-e+k-\min \{g, e-k\}$ for the sectional genus $g$ of $X$.
\end{abstract}

\section{INTRODUCTION}

Let $X \subseteq \mathbb{P}^{N}(N=n+e)$ be a nondegenerate (i.e., not contained in any hyperplane) projective variety of dimension $n \geq 1$, degree $d$, and codimension $e$ defined over an algebraically closed field $\mathbb{k}$ of arbitrary characteristic. A linear subspace $M$ of $\mathbb{P}^{N}$ is said to be secant (resp. m-secant) to $X$ if $X \cap M$ is finite with length $l(X \cap M):=\operatorname{length}\left(\mathcal{O}_{X \cap M}\right)$ positive (resp. finite with length $l(X \cap M)$ at least $\left.m\right)$. In this paper, we study the maximal length of the intersection of $X$ and a secant subspace $M$ to $X$. A secant subspace $M$ to $X$ is said to be curvilinear if each component of $X \cap M$ locally lies on a smooth curve. So a secant line to $X$ is always curvilinear. By $g$ we denote the sectional genus of $X$, i.e., the arithmetic genus of a curve obtained by taking successive hyperplane sections of $X$ (see [6, (I.2.1)]).

The purpose of this paper is to show the following:

Theorem 1. Let $X \subseteq \mathbb{P}^{N}(N=e+n)$ be a nondegenerate, projective variety of dimension $n$, degree $d$, codimension e, and sectional genus $g$. Let $M$ be a curvilinear secant linear subspace of dimension $k(1 \leq k \leq e)$ that meets $X$ on the smooth locus of $X$. Set $\ell=\min \{g, e-k\}$. Then

$$
l(X \cap M) \leq d-e+k-\ell .
$$

The bound on $l(X \cap M)$ was studied in [2, 10] and [13. In particular, the study of bounds on $l(X \cap M)$ for a line $M$ was motivated by the open conjecture on Castelnuovo-Mumford regularity (see [3, 7, §4]). This conjecture suggests that $l(X \cap M)$ would be at most $d-e+1$ for any secant line $M$. By Bertin's result in [2], this bound was proved and the boundary case was described when $X$ is smooth in $\operatorname{char}(\mathbb{k})=0$. The result of Bertin was generalized by Kwak in $\operatorname{char}(\mathbb{k})=0$ in two

Received by the editors June 1, 2007, and, in revised form, March 20, 2009.

2000 Mathematics Subject Classification. Primary 14N05, 14H45.

Key words and phrases. Secant line, secant space, sectional genus.

This work was partially supported by the Japan Society for the Promotion of Science.

(C)2009 American Mathematical Society

Reverts to public domain 28 years from publication 
directions (see [10, (1.6) and (3.6)]). First he obtained $l(X \cap M) \leq d-e+k$ for a secant $k$-dimensional subspace $M$ under the assumption that $X \cap M$ is contained in the Cohen-Macaulay locus of $X$ and that $\operatorname{dim} X=\operatorname{dim} \pi_{M}(X \backslash M)$ for the linear projection $\pi_{M}$ from $M$. Second he showed that $l(X \cap M) \leq d-e+k-1$ for a curvilinear secant subspace $M$ to a smooth $X$ of sectional genus $g \geq 1$, by describing the boundary case $l(X \cap M)=d-e+k$. The result here is a generalization of this second result of Kwak and of [13, (2.4)] where $\operatorname{char}(\mathbb{k})=0$ was assumed. On the other hand, a generalization of the first result of Kwak was studied in [13, (1.1)].

In Theorem 1, the inequality (1.1) is sharp. In fact, in [12, Theorem 1], curves with secant lines satisfying the equality in (1.1) were given (see Example 5). Also, in Proposition 6, we give a smooth curve $X$ that admits a secant $k$-plane $(k \geq 2)$ satisfying the equality in (1.1), but which for all $\lambda(1 \leq \lambda<k)$, admits no secant $\lambda$-plane satisfying the equality. Moreover, in [13, (3.4) and (3.6)], smooth scrolls with secant lines satisfying the equality in (1.1) were classified (see Remark 7).

On the other hand, in Theorem 1, the assumption that the secant space $M$ is curvilinear with $M \cap X \subseteq \operatorname{Sm} X$ is necessary to reduce the general case to the curve case. The assumption is essential to prove Lemma 3 (see Remark 4). But I know of no example of a smooth projective variety with a non-curvilinear subspace which does not satisfy (1.1).

Theorem 1 is proved by taking hyperplane sections and by reducing to the curve case, based on an idea due to Bertin 2 and Kwak [10. To this purpose, first we prove the inequality (1.1) for curves with secant lines (Proposition 2), without assuming that the secant lines meet on the smooth locus, by using a regularity bound. Next we show a Bertini-type result (Lemma 3) based on the usual Bertini Theorem, which reduces the proof of Theorem 1 to the curve case.

\section{Proof of the Main Result}

Proposition 2. Let $X \subseteq \mathbb{P}^{N}$ be a nondegenerate, projective curve of degree $d$, codimension $e$, and arithmetic genus $p_{a}(X)$. Let $L$ be a secant line to $X$. Set $\ell=\min \left\{p_{a}(X), e-1\right\}$. Then

$$
l(X \cap L) \leq d-e+1-\ell .
$$

Proof. By [7, Theorem 1.1], $X$ is $(d-e+1)$-regular, and hence the homogeneous ideal of $X$ is generated in degree $\leq d-e+1$. Thus there is no $(d-e+2)$-secant line to $X$. This implies that $l(X \cap L) \leq d-e+1$. If $p_{a}(X)=0$ or $e=1$, then $\ell=0$ and hence we have (2.1). Thus we assume that $p_{a}(X)>0$ and $e \geq 2$. Then $\ell>0$ and hence, by [11, $X$ is either $(d-e+1-\ell)$-regular or linearly normal (i.e., embedded by the complete linear system $\left|\mathcal{O}_{X}(1)\right|$ ) with $d \geq 2 p_{a}(X)+2$ and $\ell=p_{a}(X)$. In the former case, by the same argument as above, we have (2.1). In the latter case, $e-1=d-p_{a}(X)-2 \geq p_{a}(X)$ by the Riemann-Roch Theorem. Hence $\ell=p_{a}(X)$ and $d-e+1-\ell=2$. On the other hand, since $d \geq 2 p_{a}(X)+2$, by [5. Corollary 1.14], $X$ is defined by quadrics. This implies that there is no 3 -secant line to $X$, and hence we have (2.1) in the second case, too.

The following lemma based on Bertini's Theorem (see 4, (3.4.10)] or [15, (I.6.3)]) will reduce the proof of Theorem 1 to the curve case.

Lemma 3. Let $X \subseteq \mathbb{P}^{N}$ be a nondegenerate, projective variety of dimension $n$ and codimension e. Let $M$ be a curvilinear secant linear subspace of dimension $k$, 
meeting $X$ on the smooth locus of $X$. Suppose $n \geq 2$ and $e>k \geq 1$. If $H \subseteq \mathbb{P}^{N}$ is a general hyperplane containing $M$, then $X \cap H$ is irreducible, reduced, nondegenerate in $H$, and smooth at every point of $X \cap M$. Moreover, the closure $\bar{X}$ of the image $\pi_{M}(X \backslash M)$ of the linear projection $\pi_{M}$ from $M$ to $\mathbb{P}^{N-k-1}$ has dimension $n$.

Proof. Consider the linear system $\mathfrak{D}$ of hyperplane sections of $X$ by the hyperplanes containing $M$. So $X \cap H$ is a general member of $\mathfrak{D}$. First note that $X \cap H$ is smooth at every point of $X \cap M$, since a general hyperplane $H$ containing $M$ does not contain any embedded tangent space to $X$ at points of $X \cap M$ by the curvilinearity and the finiteness of $X \cap M$. Moreover $X \cap H \backslash X \cap M$ satisfies Serre's condition $S_{1}$ by a Bertini-type theorem (see [4, (3.4.6)]), since $X$ is reduced and hence satisfies $S_{1}$. Thus $X \cap H$ satisfies $S_{1}$ by the smoothness at $X \cap M$. Now we assume, for a moment, that the image of the rational map associated with $\mathfrak{D}$ has dimension $\geq 2$, and we will prove the first part of the lemma. By Bertini's Theorem (see [4. (3.4.10)] or [15, (I.6.3)]), $X \cap H$ is irreducible. Hence its dense open subset $\operatorname{Sm}(X) \cap H$ is a prime divisor or a multiple of a prime divisor on the smooth locus $\operatorname{Sm}(X)$ of $X$. Recalling that $X \cap H$ is smooth at $X \cap M$, we know that $\operatorname{Sm}(X) \cap H$ is a prime divisor on $\operatorname{Sm}(X)$ and hence that $X \cap H$ is generically reduced (i.e., $X \cap H$ satisfies Serre's condition $\left.R_{0}\right)$. Consequently $X \cap H$ satisfies $S_{1}$ and $R_{0}$, and $X \cap H$ is reduced (see [1, (VII.2.2)]). Moreover $X \cap H$ is nondegenerate in $H$ (see 4. p.116] or [8, (18.10)]).

To obtain that the image of the rational map associated with $\mathfrak{D}$ has dimension $\geq 2$, we have to show that the closure $\bar{X}$ of $\pi_{M}(X \backslash M)$ for the linear projection $\pi_{M}: \mathbb{P}^{N} \backslash M \rightarrow \mathbb{P}^{N-k-1}$ has dimension at least 2 , since $\mathfrak{D}$ defines $\pi_{M} \mid X \backslash M$. Let $\pi: X \backslash M \rightarrow \bar{X}$ be the restriction of $\pi_{M}$ to $X \backslash M$. Since the closure of a general fibre of $\pi$ lies on a $(k+1)$-dimensional linear subspace containing $M$ and since $X \cap M$ is finite, a general fibre of $\pi$ has dimension at most one and hence $\operatorname{dim} \bar{X}=n$ or $n-1$. Now suppose, to the contrary, that $\operatorname{dim} \bar{X} \leq 1$. Then $n=2$ and $\operatorname{dim} \bar{X}=1$. Note that $\bar{X}$ is nondegenerate, since $X$ is. Thus if $\operatorname{deg} \bar{X}=1$, then $\bar{X}=\mathbb{P}^{N-k-1}$ and $e=k$, a contradiction. Hence $\operatorname{deg} \bar{X} \geq 2$. Since $\bar{H}:=\pi_{M}(H \backslash M)$ is a general hyperplane of $\mathbb{P}^{N-k-1}$ by the generality of $H \supseteq M$, the intersection $\bar{X} \cap \bar{H}$ consists of $\bar{d}$ distinct points $\bar{x}_{1}, \ldots, \bar{x}_{\bar{d}}$ for $\bar{d}:=\operatorname{deg} \bar{X} \geq 2$ such that $\bar{x}_{i}=\pi_{M}\left(x_{i}\right)$ for some $x_{i} \in X \backslash M$, by Bézout's Theorem. Hence $X \cap H=C_{\bar{x}_{1}} \cup \cdots \cup C_{\bar{x}_{\bar{d}}}$ for the closure $C_{\bar{x}_{i}}$ of the fibre $\pi^{-1}\left(\bar{x}_{i}\right)$. On the other hand, $X \cap H$ is connected by a Lefschetz type theorem [9, (II.6.2)]. But $C_{\bar{x}_{1}}, \ldots, C_{\bar{x}_{\bar{d}}}$ meet only at $X \cap M$, since $\left\langle M, x_{i}\right\rangle \cap\left\langle M, x_{j}\right\rangle=M$ for $i \neq j$. This contradicts the smoothness of $X \cap H$ at $X \cap M$. Thus $\operatorname{dim} \bar{X} \geq 2$, as required.

To show the second part of the lemma, $\operatorname{dim} \bar{X}=\operatorname{dim} X$, by taking the hyperplane section $X \cap H$ for a general hyperplane $H$ containing $M$ and applying the first part, we may assume that $\operatorname{dim} X=2$ and have to show that $\operatorname{dim} \bar{X}=2$. This has already been done in the paragraph above.

Remark 4. We cannot relax the assumption of Lemma 3: If $X \cap M$ is not curvilinear or if $X$ is not smooth at $X \cap M$, then the conclusion of Lemma 3 is not true in general (see [13, Example 2.2]).

Proof of Theorem 1. By Lemma 3, upon taking general hyperplanes $H_{i}(i=1, \ldots$, $n-1)$ containing $M$ and replacing $X$ by $X \cap H_{1} \cap \cdots \cap H_{n-1}$, we may assume that $X \subseteq \mathbb{P}^{N}(N=e+1)$ is a nondegenerate projective curve of degree $d$, arithmetic genus $p_{a}(X)=g$, and codimension $e$, with a secant $k$-space $M$ meeting it on the 
smooth locus of $X$. Let $\Lambda$ be a general $(k-2)$-dimensional subspace of $M$. So $\Lambda \cap\left\langle x_{i}, x_{j}\right\rangle=\emptyset$ and $\Lambda \cap T_{x_{i}}(X)=\emptyset$ for all $x_{i} \neq x_{j} \in X \cap M$, and in particular $X \cap \Lambda=\emptyset$. Consider the linear projection $\pi_{\Lambda}: \mathbb{P}^{N} \backslash \Lambda \rightarrow \mathbb{P}^{N-k+1}$ from $\Lambda$. Set $\bar{X}=\pi_{\Lambda}(X)$ and $\bar{M}=\pi_{\Lambda}(M \backslash \Lambda)$. At each point of $\bar{X} \cap \bar{M}$, the induced morphism $\pi=\pi_{\Lambda} \mid X: X \rightarrow \bar{X}$ is an isomorphism by the choice of $\Lambda$. Thus $\pi$ is a birational morphism, and $\bar{M}$ is a secant line to $\bar{X}$ with $l(X \cap M)=l(\bar{X} \cap \bar{M})$. Moreover $\bar{X}$ is a nondegenerate projective curve of $\operatorname{deg} \bar{X}=\operatorname{deg} X$ and codimension $e-k+1$, with $p_{a}(\bar{X}) \geq p_{a}(X)=g$. Applying Proposition 2 to $\bar{X}$ and $\bar{M}$, we have

$$
l(\bar{X} \cap \bar{M}) \leq d-(e-k+1)+1-\min \left\{p_{a}(\bar{X}),(e-k+1)-1\right\} .
$$

Consequently we have (1.1).

\section{EXAMPles OF THE BOUNDARY CASE}

In this section, we will give examples of projective curves that admit secant $k$-planes attaining the equality in Theorem 1 .

Example 5 ([12, Theorem 1]). There exists a smooth projective curve $X \subseteq \mathbb{P}^{N}$ of degree $d$ and genus $g$ with secant line $L$ satisfying one of the following conditions.

(1) $N \geq g+2, d \geq N+g+1$ and $l(X \cap L)=d-N+2-g$. In this case $\ell=\min \{g, N-2\}=g$.

(2) $N=g+1 \geq 3, d \geq 2 g+2$ and $l(X \cap L)=d-N+2-(g-1)=$ $d-N+2-(N-2)$. In this case $\ell=\min \{g, N-2\}=N-2=g-1$.

(3) $X$ is hyperelliptic, $g+1>N \geq 3, d \geq 2 g+2$ and $l(X \cap L)=d-N+2-$ $(N-2) \geq 6$. In this case $\ell=\min \{g, N-2\}=N-2$.

In the next proposition, by an elementary argument similar to the proof of [12, Theorem 1], we will give a smooth curve $X$ that admits a secant $k$-plane $(k \geq 2)$ reaching the bound in Theorem 1 and Proposition 2, but which for all $\lambda(1 \leq \lambda<k)$ does not admit any secant $\lambda$-plane reaching the bound.

Proposition 6. Let $g, k, N$ and $d$ be integers with

$$
g \geq 0, k \geq 2, N \geq g+2 k+1 \text { and } 2(N-k) \geq d \geq g+N+1 .
$$

There exists a smooth, nondegenerate, projective curve $X \subseteq \mathbb{P}^{N}$ of genus $g$ that admits a k-plane $M$ with $l(X \cap M)=d-N+1+k-g$, but which for all $\lambda$ $(1 \leq \lambda<k)$ admits no $\lambda$-plane $L$ with $l(X \cap L)=d-N+1+\lambda-g$.

Proof. If we set $t:=d-g-N-1$, it is easy to see that giving the integers $g, k$, $N$ and $d$ in (6.0) is equivalent to giving integers $g, k, t$, and $d$ such that

$$
g \geq 0, k \geq 2, t \geq 0, \text { and } d \geq 2 g+2 k+2 t+2 .
$$

Thus setting $b:=k+t+1$, we will construct an embedding of a smooth projective curve $X$ into $\mathbb{P}^{N}(N=d-g-t-1)$, where $X$ is nondegenerate in $\mathbb{P}^{N}$, of degree $d$ and genus $g$, and such that it admits a $k$-plane $M$ with $l(X \cap M)=d-(N-$ $1)+k-g=b+1$ but for all $\lambda(1 \leq \lambda<k)$ admits no $\lambda$-plane $L$ with $l(X \cap L)=$ $d-(N-1)+\lambda-g=t+\lambda+2$. To this purpose, let $X$ be a smooth projective curve of genus $g$, and first embed $X$ into $\mathbb{P}^{d-g}$ by the complete linear system associated with a line bundle $\mathcal{O}_{X}(1)$ of degree $d$. Note that $\operatorname{dim} H^{0}\left(\mathcal{O}_{X}(1)\right)=d-g+1$ since $d \geq 2 g+1$. To obtain the required embedding of $X$, we will project $X$ down to $\mathbb{P}^{N}$ from a suitable $t$-dimensional linear space $\Lambda$. 
Before showing how to choose $\Lambda$, we will look at the linear span of divisors on $X$ in $\mathbb{P}^{d-g}$. For a subset $Z \subseteq \mathbb{P}^{d-g}$, the linear span $\langle Z\rangle$ of $Z$ in $\mathbb{P}^{d-g}$ is the intersection of all hyperplanes of $\mathbb{P}^{d-\bar{g}}$ that contain $Z$. Thus for an effective divisor $D$ on $X,\langle D\rangle$ is defined by $H^{0}\left(\mathcal{O}_{X}(1) \otimes \mathcal{O}_{X}(-D)\right)$. In this case, it is clear by the Riemann-Roch Theorem that

$$
\operatorname{dim}\langle D\rangle=\operatorname{deg} D-1 \quad \text { if } \quad \operatorname{deg} D \leq 2 b+1,
$$

since $d \geq 2 g+2 b$ by assumption. On the other hand, for effective divisors $D_{1}$ and $D_{2}$ on $X$, we define $D_{1} \vee D_{2}$ to be the smallest effective divisor $D^{\prime}$ with $D^{\prime} \geq D_{1}$ and $D^{\prime} \geq D_{2}$, where we say that $D^{\prime} \geq D_{1}$ if $D^{\prime}-D_{1}$ is effective, and we also define $D_{1} \wedge D_{2}$ to be the biggest effective divisor $D^{\prime \prime}$ with $D^{\prime \prime} \leq D_{1}$ and $D^{\prime \prime} \leq D_{2}$. In this case, $\operatorname{deg} D_{1} \vee D_{2}+\operatorname{deg} D_{1} \wedge D_{2}=\operatorname{deg} D_{1}+\operatorname{deg} D_{2}$. With this notation, we have

$$
\left\langle\left\langle D_{1}\right\rangle \cup\left\langle D_{2}\right\rangle\right\rangle=\left\langle D_{1} \vee D_{2}\right\rangle,
$$

since as subspaces of $H^{0}\left(\mathcal{O}_{X}(1)\right)$,

$H^{0}\left(\mathcal{O}_{X}(1) \otimes \mathcal{O}_{X}\left(-D_{1}\right)\right) \cap H^{0}\left(\mathcal{O}_{X}(1) \otimes \mathcal{O}_{X}\left(-D_{2}\right)\right)=H^{0}\left(\mathcal{O}_{X}(1) \otimes \mathcal{O}_{X}\left(-\left(D_{1} \vee D_{2}\right)\right)\right)$.

Take any points $x_{1}, \ldots, x_{b+1} \in X$ and set $D:=x_{1}+\cdots+x_{b+1}$. By (6.1), we have $\operatorname{dim}\langle D\rangle=b$. Let $\Lambda \subseteq\langle D\rangle$ be a $t$-plane such that

$$
\Lambda \nsubseteq \bigcup_{0 \leq D^{\prime} \leq D, \operatorname{deg} D^{\prime}=b}\left\langle D^{\prime}\right\rangle \text { and } \Lambda \cap \bigcup_{0 \leq D^{\prime \prime} \leq D, \operatorname{deg} D^{\prime \prime}=2}\left\langle D^{\prime \prime}\right\rangle=\emptyset .
$$

We claim that for any effective divisors $E$ and $B$ with $\operatorname{deg} E=t+\lambda+2(\leq b)$ and $\operatorname{deg} B=2$,

$$
\Lambda \nsubseteq\langle E\rangle \quad \text { and } \quad \Lambda \cap\langle B\rangle=\emptyset .
$$

Assuming the claim, we will complete the proof. Let $\pi_{\Lambda}: \mathbb{P}^{d-g} \rightarrow \rightarrow \mathbb{P}^{N}$ be the linear projection from $\Lambda$. From the latter condition of (6.4), it follows that $X$ is isomorphic onto its image $\pi_{\Lambda}(X)$. Clearly the image $M:=\pi_{\Lambda}(\langle D\rangle)$ is a linear subspace of dimension $b-t-1=k$ such that $l\left(\pi_{\Lambda}(X) \cap M\right)=b+1$. Suppose to the contrary that there exists a $\lambda$-plane $L(1 \leq \lambda<k)$ with $l\left(\pi_{\Lambda}(X) \cap L\right)=t+\lambda+2$. Then $\pi_{\Lambda}(X) \cap L$ can be seen an effective divisor $E$ on $X$ by the isomorphism $X \cong \pi_{\Lambda}(X)$. In this case, $\pi_{\Lambda}(\langle E\rangle) \subseteq L$ and $\operatorname{dim}\langle E\rangle=t+\lambda+1$ by (6.1). Therefore $\Lambda$ must be contained in $\langle E\rangle \subseteq \mathbb{P}^{d-g}$, which contradicts (6.4).

Finally we prove the claim (6.4). To prove the first part, suppose to the contrary that $\Lambda \subseteq\langle E\rangle$ for an effective divisor $E$ of $\operatorname{deg} E=h \leq b$. If necessary, upon replacing $E$ by an effective divisor less than $E$ we may assume that $\Lambda \subseteq E$ but $\Lambda \nsubseteq\left\langle E^{\prime}\right\rangle$ for any effective divisor $E^{\prime} \leq E$ of degree $h-1$. Thus $D \wedge E \neq E$ by the first part of (6.3). Let $E_{1}$ be an effective divisor with $D \wedge E \leq E_{1}<E$ and $\operatorname{deg} E_{1}=\operatorname{deg} E-1$. Hence $\Lambda \nsubseteq\left\langle E_{1}\right\rangle$. Since $D \wedge E=D \wedge E_{1}$, we have $\operatorname{deg}(D \vee E)=\operatorname{deg}\left(D \vee E_{1}\right)+1 \leq 2 b+1$, and hence $\langle D \vee E\rangle \neq\left\langle D \vee E_{1}\right\rangle$ by (6.1). On the other hand, $\Lambda \subseteq\langle E\rangle$ but $\Lambda \nsubseteq\left\langle E_{1}\right\rangle$ by the choice of $E$ and $E_{1}$, and hence $\left\langle\Lambda \cup\left\langle E_{1}\right\rangle\right\rangle=\langle E\rangle$. This implies that $\langle\langle D\rangle \cup\langle E\rangle\rangle=\left\langle\langle D\rangle \cup \Lambda \cup\left\langle E_{1}\right\rangle\right\rangle=\left\langle\langle D\rangle \cup\left\langle E_{1}\right\rangle\right\rangle$, which contradicts $\langle D \vee E\rangle \neq\left\langle D \vee E_{1}\right\rangle$ by $(6.2)$.

To prove the second part of (6.4), suppose to the contrary that $\Lambda \cap\langle B\rangle \neq \emptyset$ for an effective divisor $B$ of $\operatorname{deg} B=2$. Then $\operatorname{dim}\langle\langle D\rangle \cup\langle B\rangle\rangle \leq b+1$ since $\Lambda \subseteq\langle D\rangle$. Hence $\operatorname{deg}(D \vee B) \leq b+1$ by (6.1) and (6.2). Thus there exists a point $y \in \operatorname{Supp} D \cap \operatorname{Supp} B$. By the choice of $\Lambda$ in (6.3), we have $y \notin \Lambda$, and hence $\langle\Lambda \cup\{y\}\rangle=\langle\Lambda \cup\langle B\rangle\rangle$ by comparing the dimension. This implies that 
$\langle\langle D\rangle \cup\langle B\rangle\rangle=\langle\langle D\rangle \cup \Lambda \cup\langle B\rangle\rangle=\langle\langle D\rangle \cup \Lambda \cup\{y\}\rangle=\langle D\rangle$. Hence $D \geq B$ by (6.1) and (6.2). This contradicts the choice of $\Lambda$ in (6.3).

Remark 7. In [13, (3.4) and (3.6)], scrolls with secant lines satisfying the equality in (1.1) were classified. Also, in [14, smooth Del Pezzo varieties with secant lines satisfying the equality in (1.1) were classified. But much is not known yet for $n \geq 2$ and $k \geq 2$.

\section{REFERENCES}

1. A. Altman and S. Kleiman, Introduction to Grothendieck duality theory, Lecture Notes in Math., 146, Springer-Verlag, 1970. MR0274461 (43:224)

2. M. A. Bertin, On the regularity of varieties having an extremal secant line, J. Reine Angew. Math. 545 (2002), 167-181. MR.1896101(2003h:14078)

3. D. Eisenbud and S. Goto, Linear free resolutions and minimal multiplicity, J. Algebra 88 (1984), 89-133. MR741934 (85f:13023)

4. H. Flenner, L. O'Carroll, and W. Vogel, Joins and intersections, Springer Monographs in Mathematics, Springer-Verlag, 1999. MR1724388 (2001b:14010)

5. T. Fujita, Defining equations for certain types of polarized varieties, Complex analysis and algebraic geometry, Cambridge University Press, 1977, pp. 165-173. MR.0437533 (55:10457)

6. T. Fujita, Classification theories of polarized varieties, London Mathematical Society Lecture Note Series, 155, Cambridge University Press, 1990. MR1162108 (93e:14009)

7. L. Gruson, R. Lazarsfeld, and C. Peskine, On a theorem of Castelnuovo, and the equations defining space curves, Invent. Math. 72 (1983), 491-506. MR704401 (85g:14033)

8. J. Harris, Algebraic geometry, Graduate Texts in Mathematics, 133, Springer-Verlag, 1992. MR:1182558 (93j:14001)

9. R. Hartshone, Ample subvarieties of algebraic varieties, Lecture Notes in Mathematics, 156, Springer-Verlag, 1970. MR0282977 (44:211)

10. S. Kwak, Smooth projective varieties with extremal or next to extremal curvilinear secant subspaces, Trans. Amer. Math. Soc. 357 (2005), 3553-3566. MR2146638 (2006e:14072)

11. A. Noma, A bound on the Castelnuovo-Mumford regularity for curves, Math. Ann. 322 (2002), 69-74. MR:1883389 (2002k:14046)

12. A. Noma, Castelnuovo-Mumford regularity of nonhyperelliptic curves, Arch. Math. (Basel) 83, no. 1 (2004), 23-26. MR2079822 (2005c:14039)

13. A. Noma, Multisecant lines to projective varieties, Projective varieties with unexpected properties, Walter de Gruyter, 2005, pp. 349-359. MR2202263 (2006k:14099)

14. A. Noma, Multisecant lines to smooth Del Pezzo varieties, preprint, 2007.

15. O. Zariski, Introduction to the problem of minimal models in the theory of algebraic surfaces, Publ. of the Math. Soc. of Japan, no. 4, The Mathematical Society of Japan, Tokyo, 1958. MR0097403 (20:3872)

Department of Mathematics, Faculty of Education and Human Sciences, Yokohama NATiOnal University, Yokohama 240-8501, Japan

E-mail address: noma@edhs.ynu.ac.jp 